\title{
Hybrid Dispersion Laser Scanner
}

\author{
K. Goda', ${ }^{1,3}$, A. Mahjoubfar ${ }^{1,2}$, C. Wang ' , A. Fard' ${ }^{1,2}$, J. Adam ', D. R. Gossett ${ }^{2,3}$, A. Ayazi ${ }^{1}$, E. Sollier ${ }^{3}$, \\ O. Malik' , E. Chen' ', Y. Liu' ', R. Brown', N. Sarkhosh', D. Di Carlo, \& B. Jalali1, 2,3,4
}

SUBJECT AREAS:

IMAGING

ULTRAFAST PHOTONICS

FIBRE OPTICS AND OPTICAL COMMUNICATIONS

BIOPHOTONICS

Received

21 March 2012

Accepted

21 May 2012

Published

8 June 2012

Correspondence and requests for materials should be addressed to K.G. Igoda@ee.ucla. edu)
'Department of Electrical Engineering, University of California, Los Angeles, California 90095, ${ }^{2}$ California NanoSystems Institute, Los Angeles, California 90095, ${ }^{3}$ Department of Bioengineering, University of California, Los Angeles, California 90095,

${ }^{4}$ Department of Surgery, David Geffen School of Medicine, University of California, Los Angeles, California 90095.

Laser scanning technology is one of the most integral parts of today's scientific research, manufacturing, defense, and biomedicine. In many applications, high-speed scanning capability is essential for scanning a large area in a short time and multi-dimensional sensing of moving objects and dynamical processes with fine temporal resolution. Unfortunately, conventional laser scanners are often too slow, resulting in limited precision and utility. Here we present a new type of laser scanner that offers $\sim 1,000$ times higher scan rates than conventional state-of-the-art scanners. This method employs spatial dispersion of temporally stretched broadband optical pulses onto the target, enabling inertia-free laser scans at unprecedented scan rates of nearly $100 \mathrm{MHz}$ at $800 \mathrm{~nm}$. To show our scanner's broad utility, we use it to demonstrate unique and previously difficult-to-achieve capabilities in imaging, surface vibrometry, and flow cytometry at a record $2 \mathrm{D}$ raster scan rate of more than $100 \mathrm{kHz}$ with 27,000 resolvable points.

aser scanning technology is one of the most integral parts of today's scientific research ${ }^{1-8}$, manufacturing ${ }^{1-3,9-13}$, defense ${ }^{1,2,9,1,13}$, and biomedicine $e^{1,4-7,14-16}$. For sensing and imaging methods based on laser scanners ${ }^{1-16}$, the ability to scan at high speeds is crucial for multi-dimensional evaluation of moving objects and dynamical processes. Here low scan rates result in motion blur and failure to capture fast transient information. Applications that require high scan rates (typically $\sim 1 \mathrm{kHz}$ or higher in $2 \mathrm{D}$ ) include identification of missiles and aircrafts via light detection and ranging (LIDAR) ${ }^{1-3,8,9,12,13}$, non-destructive inspection of acoustic instruments ${ }^{10}$, structural dynamics $^{10,12}$, and microelectromechanical systems $(\mathrm{MEMS})^{11,12}$ via laser-scanning surface vibrometry ${ }^{1,1,12}$, and observation of biomechanical motility ${ }^{4}$, cellular network dynamics ${ }^{5}$, and neural activity ${ }^{6,7}$ via laser-scanning confocal and multi-photon microscopy ${ }^{1,3,6,7}$. The central requirement for sensing and imaging of such events in real time is the temporal resolution shorter than the time scale of changes in the dynamical process. Furthermore, highspeed scanning capability is also important for high-throughput applications that require scanning a large area or many objects in a short period of time, such as geographical survey ${ }^{2}$, atmospheric science ${ }^{2,8}$, machine vision ${ }^{1,2,13}$, laser TV systems ${ }^{17}$, surface profilometry in the semiconductor industry ${ }^{1,3}$, and endoscopy and cytometry for medical diagnosis ${ }^{14-16}$.

Various types of laser scanning technology have been developed for higher scan rates in the past few decades ${ }^{1}$. The most commonly used for beam steering are mechanically scanning mirrors known as galvanometric mirrors $^{1,2,18}$. Unfortunately, the galvanometric mirrors including MEMS scanners ${ }^{18}$ are slow due to inertia and provide linear scan rates only up to $\sim 10 \mathrm{kHz}$, resulting in aggregate $2 \mathrm{D}$ scan rates of $\sim 100 \mathrm{~Hz}$ at most ${ }^{1,18}$. Another common type of laser scanners is acousto-optic deflectors (AODs) which provide $\sim 10$ times higher scan rates than the traditional galvanometric mirrors by virtue of their acoustic frequency dependent diffraction (i.e., $\sim 100 \mathrm{kHz}$ in $1 \mathrm{D}$ and $\sim 1 \mathrm{kHz}$ in $2 \mathrm{D})^{1,19}$. In addition, a combination of a frequency-tunable laser and diffractive optics has recently been found to operate as a laser scanner at scan rates comparable to AODs ${ }^{20,21}$. To the best of our knowledge, these mechanisms provide the highest scan rates possible to date.

In this Article, we propose and demonstrate a new method for laser scanning that enables ultrafast inertia-free scans in the industrially and biomedically important spectral band (i.e., $\sim 800 \mathrm{~nm}$ ) at $\sim 1000$ times higher scan rates than conventional laser scanners. This approach is based on spatial dispersion of a broadband optical pulse onto the target with the pulse being linearly chirped by temporal dispersion so that each frequency component of the pulse arrives at a different set of spatial coordinates on the target at a different time. The scanner's ability to scan without the need for mechanical and active electronic components eliminates the speed bottleneck that exists in galvanometric mirrors and other techniques and hence enables ultrafast scanning at the rate equivalent to the laser's pulse repetition rate (typically $10-100 \mathrm{MHz}$ ). As a proof-of-principle demonstration, we show 1D line scans at more than $90 \mathrm{MHz}$ and 2D raster scans at more than $100 \mathrm{kHz}$ with 27,000 resolvable points and a dwell time of less than 60 ps. Furthermore, to show the broad utility of our method, we use it to demonstrate unique and 


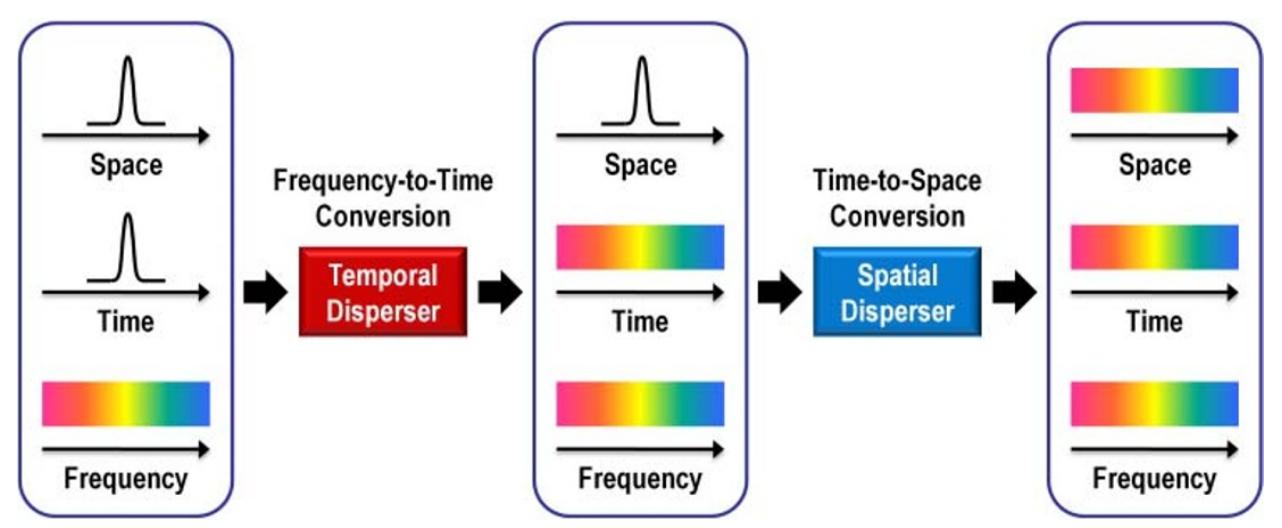

Figure $1 \mid$ Concept of the HDLS. The operation of the HDLS consists of two steps: frequency-to-time conversion and time-to-space conversion. First, the broadband spectrum of an optical pulse is mapped into a temporal waveform by temporal dispersion. Second, the temporal waveform is converted into a $1 \mathrm{D}$ rainbow pulse in space by spatial dispersion. The resultant $1 \mathrm{D}$ rainbow pulse is incident onto an object, performing a $1 \mathrm{D}$ line scan. Combining the two steps, the spectrum of the pulse is mapped into space, resulting in each frequency component of the spectrum illuminating different spatial coordinates on the target at a different time. Pulses are repeated for repetitive scans at a rate equivalent to the pulse repetition rate.

previously difficult-to-achieve capabilities in imaging, surface vibrometry, and flow cytometry by virtue of its ultrahigh scan rates.

\section{Results}

Principle of the laser scanner. The concept of our laser scanner, which we refer to as the hybrid dispersion laser scanner (HDLS), is shown in Figure 1. Its operation consists of two steps: frequency-totime conversion and time-to-space conversion. First, the broadband spectrum of an optical pulse is mapped into a temporal waveform by temporal dispersion. This step is known as wavelength-to-time mapping $^{22-24}$ or dispersive Fourier transformation ${ }^{25-29}$ and is performed using large group-velocity dispersion (GVD) in dispersive fibers or chirped fiber Bragg gratings. Second, the temporal waveform is converted by spatial dispersion into a $1 \mathrm{D}$ rainbow pulse in space. The spatial disperser may be one or more prisms or diffraction gratings. The resultant $1 \mathrm{D}$ rainbow pulse is incident onto an object, performing a $1 \mathrm{D}$ line scan. Combining the two steps, the spectrum of the pulse is mapped into space, resulting in each frequency component of the spectrum illuminating a different set of spatial coordinates on the target at a different time in series. The reflected, transmitted, or fluorescence light from the target can be detected by a single photodetector (e.g., a photodiode, photomultiplier tube, or avalanche photodetector). Pulses are repeated for repetitive scans at a rate equivalent to the pulse repetition rate. For $2 \mathrm{D}$ raster scans, a complementary scanner (i.e., the slow axis scanner) is used to scan the HDLS rainbow beam along the second planar dimension.

To maximize the practical utility of the HDLS, we design and implement it in the spectral range important for industrial and biomedical settings (i.e., $\sim 800 \mathrm{~nm}$ ). For this purpose, we perform dispersive Fourier transformation in the $800 \mathrm{~nm}$ spectral band for the first time. Previously, dispersive Fourier transformation has been restricted to the fiber-optic communication band centered at $\sim 1550 \mathrm{~nm}$ due to the commercial unavailability of dispersive fibers with high dispersion-to-loss ratio outside the $\sim 1550 \mathrm{~nm}$ band $^{22-25,27-29}$. On the contrary, shorter wavelengths are desirable since the spectral resolution limited by diffractive elements (or equivalently the HDLS's number of resolvable points) improves with shorter wavelengths (See Methods). Also, the $800 \mathrm{~nm}$ spectral band is suitable for biomedical applications as it enables larger penetration depths in tissue and reduction in auto-fluorescence ${ }^{30}$. To demonstrate dispersive Fourier transformation in this spectral band, we employ a single-mode fiber with a smaller effective mode field diameter $(4 \mu \mathrm{m})$ than conventional fibers in the wavelength operation range (typically 5-6 $\mu \mathrm{m}$ ). The fiber, hence, provides increased contribution of waveguide dispersion to the total chromatic dispersion of the fiber. Our chromatic dispersion measurement indicates a large GVD of $-123 \mathrm{ps} / \mathrm{nm} / \mathrm{km}$ (Figure 2), enabling dispersive Fourier transformation in this spectral band.

To demonstrate the HDLS, we constructed the apparatus shown in Figure 3. The optical source is a Ti:Sapphire femtosecond pulse laser that generates a train of broadband pulses at a pulse repetition rate of 90.8 MHz. Before entering the dispersive fiber, the pulses are prechirped by a prism-based pulse stretcher to avoid undesirable nonlinear interactions in the fiber. The pre-chirped pulses then enter the dispersive fiber in which the spectrum of each pulse is stretched into a temporal waveform due to the GVD of the fiber $(-583 \mathrm{ps} / \mathrm{nm})$. The AOD scans the pulses in the vertical direction with 132 resolvable points at a scan rate of $105.4 \mathrm{kHz}$ (See Methods). A pair of diffraction gratings with 2200 lines/mm spatially disperses the pulses in the horizontal direction, producing 202 resolvable subpulses (See Methods). When combined, the temporally dispersed pulses are scanned over the target by the gratings in the horizontal direction at $90.8 \mathrm{MHz}$ (equivalent to the pulse repetition rate) and by the AOD in the vertical direction at $105.4 \mathrm{kHz}$ for complete $2 \mathrm{D}$ raster scans.

Ultrafast reflection imaging with the laser scanner. To demonstrate the HDLS's one-to-one mapping between frequency, time, and space, we used it to conduct reflection imaging in real time. Figure $4 \mathrm{a}$ compares a CCD image of a test target and a digitally reconstructed image of the target scanned by the HDLS. The HDLS image was reconstructed by mapping the reflection from the target measured by a photodetector into a $2 \mathrm{D}$ matrix using a separately measured pulse train and AOD driver signal. Figure $4 \mathrm{~b}$ shows the performance of dispersive Fourier transformation in the $800 \mathrm{~nm}$ spectral range, validating one-to-one mapping between frequency (wavelength) and time and hence between time and space. The captured image clearly shows the word "UCLA," firmly establishing the HDLSbased imaging.

Ultrafast sub-nanometer-scale surface vibrometry with the laser scanner. To further show the utility of the HDLS, we used it to perform real-time observation of fast nanomechanical surface vibrations with sub-nanometer axial resolution. As stated above, high-speed surface vibrometry is important for non-destructive diagnosis of mechanical components, but its real-time operation has been difficult due to the speed limitation of scanners in conventional laserscanning surface vibrometers. While stroboscopic imaging can be used to evaluate the dynamics of mechanical systems faster than the scan rate of the imager ${ }^{31}$, it requires their motion to be repetitive (i.e., vibrating, rotating, oscillating, or reciprocating), unable to monitor random or non-repetitive dynamics in the form of transient rogue 

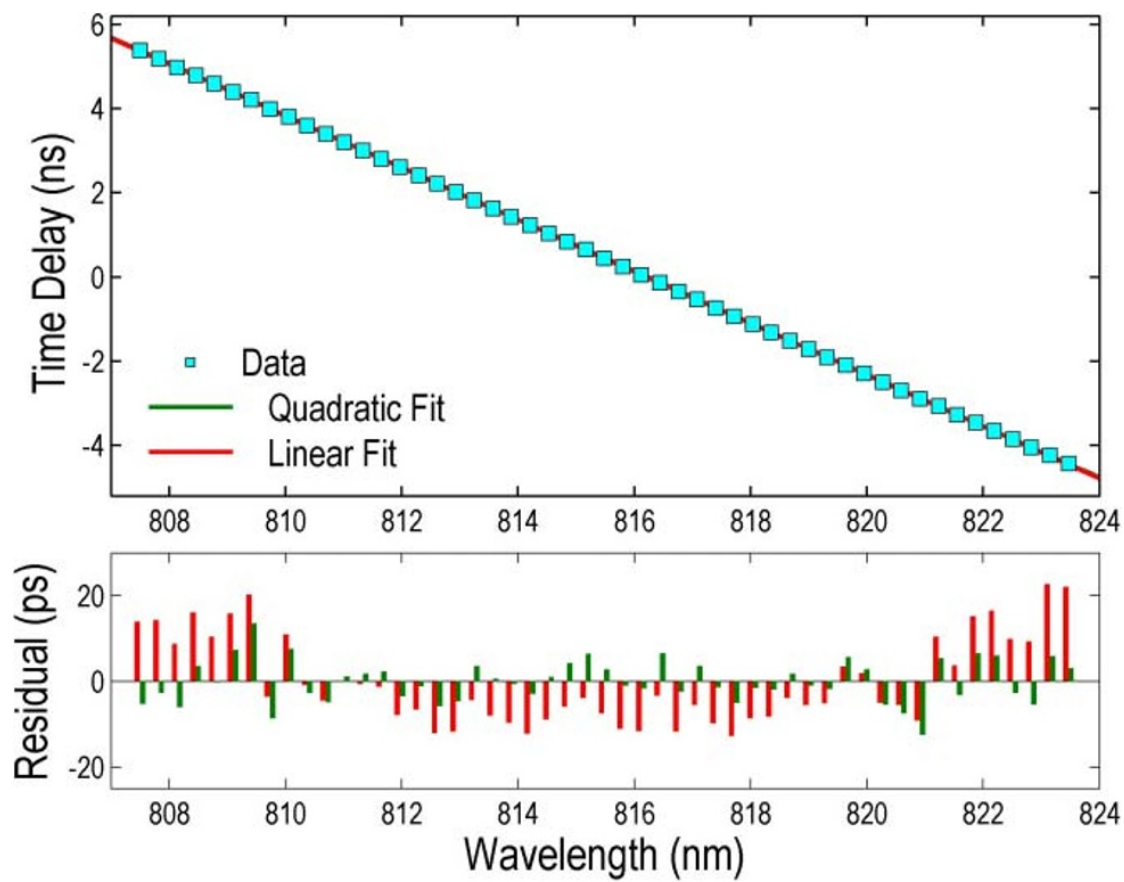

Figure $2 \mid$ Chromatic dispersion of the dispersive fiber. To demonstrate dispersive Fourier transformation in the $\sim 800 \mathrm{~nm}$ spectral band, a single-mode fiber with a smaller effective mode area than conventional fibers in the wavelength operation range is employed. Consequently, the fiber provides increased contribution of waveguide dispersion to the total chromatic dispersion of the fiber (as large as $-123 \mathrm{ps} / \mathrm{nm} / \mathrm{km} \mathrm{GVD)}$ ), enabling dispersive Fourier transformation in this spectral range as indicated by the linear one-to-one relation between wavelength (frequency) and time as well as the small residuals between the data and fit functions.

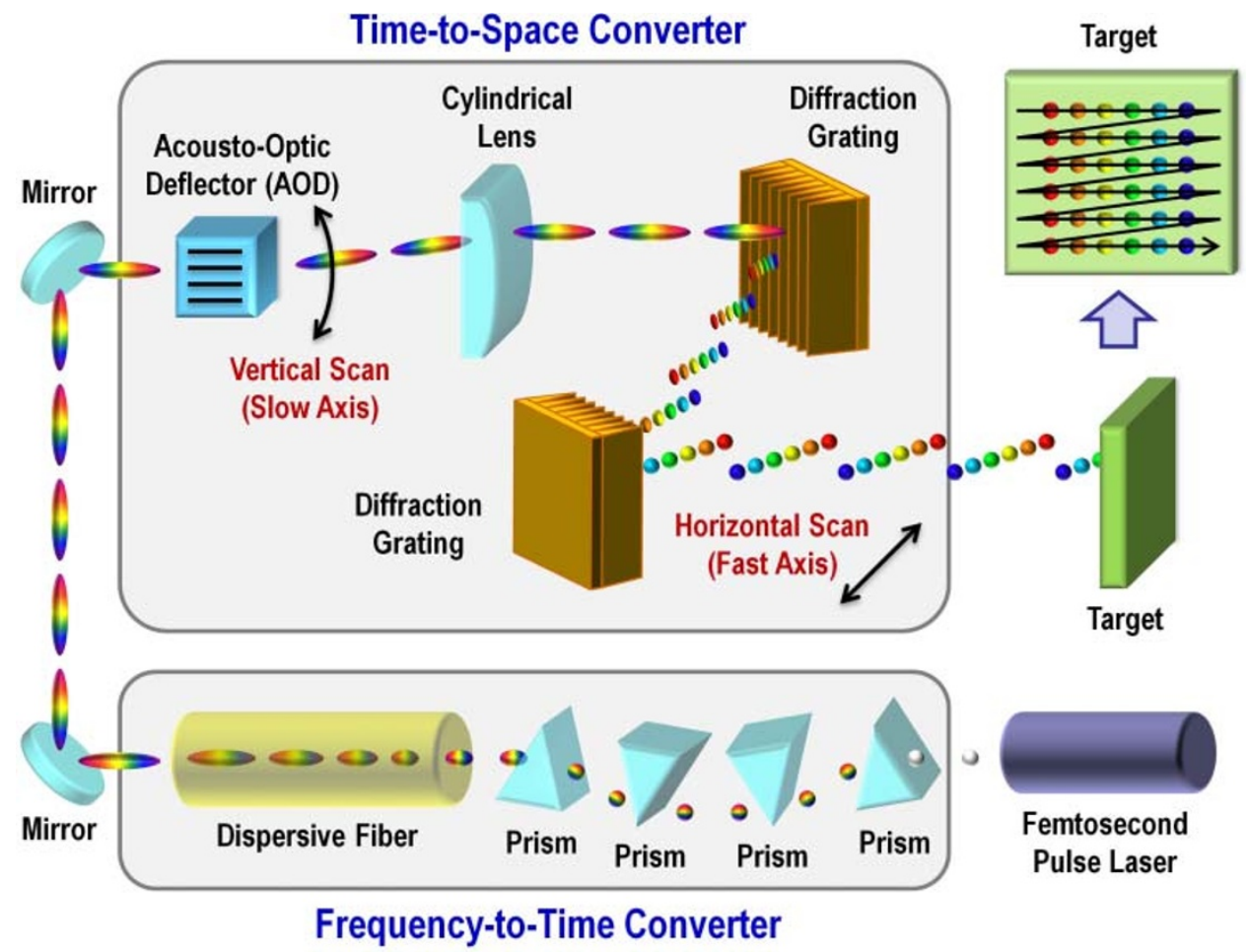

Figure $3 \mid$ Schematic of the HDLS. The optical source is a Ti:Sapphire femtosecond pulse laser that generates a broadband pulse train at $90.8 \mathrm{MHz}$ repetition rate. The pulses are pre-chirped by the prism-based pulse stretcher and enter the dispersive fiber in which the spectrum of each pulse is mapped into the time domain by the GVD of the fiber. A pair of diffraction gratings maps the pulses into space in the horizontal direction, producing 202 subpulses which arrive at different spatial coordinates on the target at different times. Consequently, 1D line scans are performed in the horizontal direction at $90.8 \mathrm{MHz}$. The AOD scans the pulses in the vertical direction at $105.4 \mathrm{kHz}$ with 132 resolvable points, resulting in the aggregate resolution of $202 \times 132$ (horizontal x vertical) (See Methods). The cylindrical lens collimates the pulses deflected at different angles. The colors of the subpulses in the figure are only for illustrative purposes and do not represent the real wavelengths. 

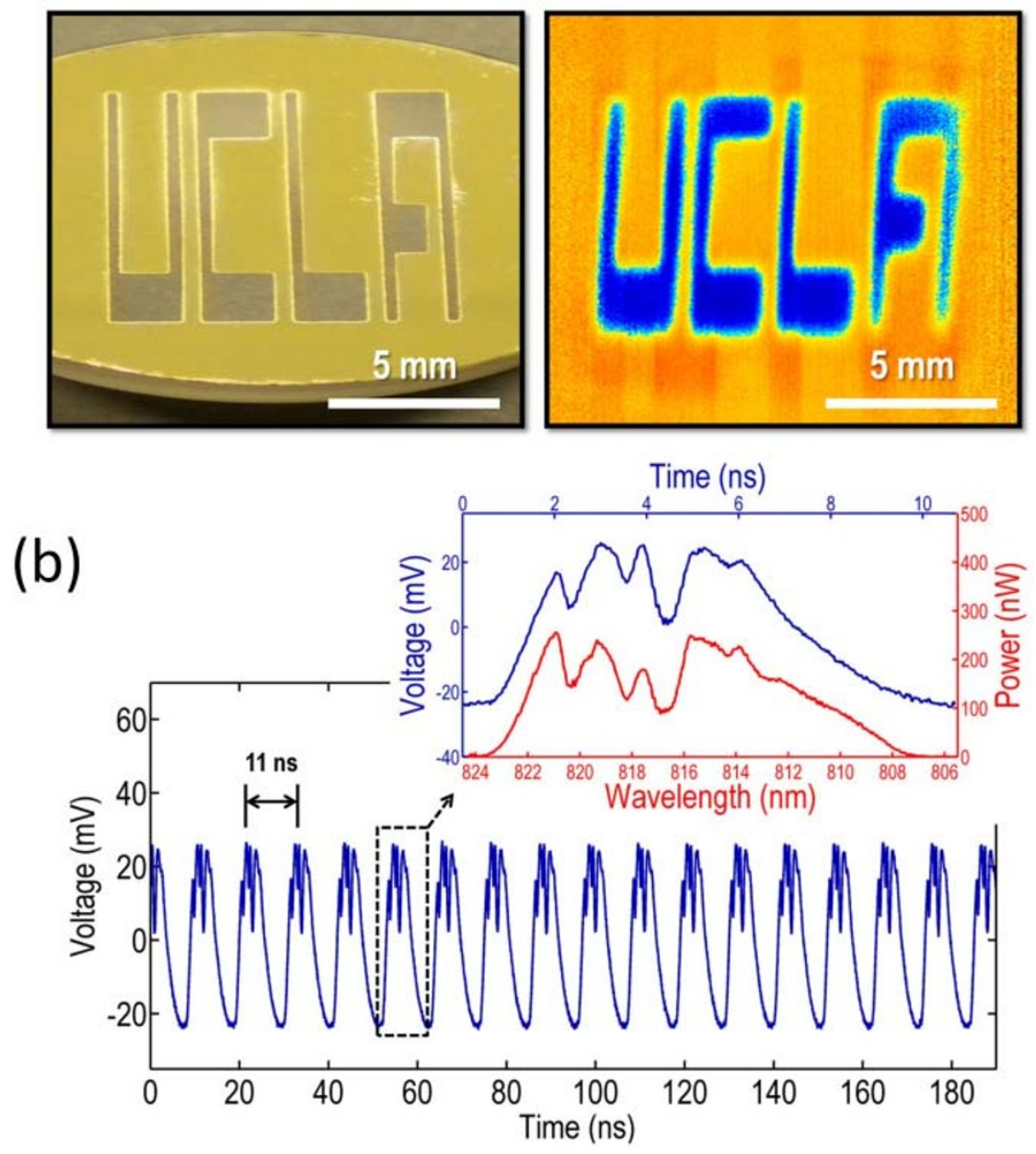

Figure $4 \mid$ Imaging with the HDLS. (a) Comparison between a CCD image of the test target (left) and a digitally reconstructed image of the target scanned by the HDLS (right). The target consists of the non-reflective word "UCLA" on top of a reflective substrate and was scanned by the HDLS at 90.8 MHz in 1D and 105.4 kHz in 2D for image acquisition. The word "UCLA" appears evident (with the reddish and bluish colors indicating high and low reflectivities, respectively). The number of pixels in the target image is $510 \times 861$ (horizontal x vertical) (See Methods). The distortion on the right hand side of the letter " $\mathrm{A}$ " is due to the reduced diffraction efficiency of the AOD at a large deflection angle near the edge of the field of view.

(b) Horizontal line scans (pulses) at different vertical coordinates (different deflection angles of the AOD) with a time interval of $11 \mathrm{ns,} \mathrm{corresponding} \mathrm{to}$ the pulse repetition rate of $90.8 \mathrm{MHz}$. The inset shows one-to-one mapping between the reflection signal from the target measured by the photodetector and the spectrum of the signal measured by a conventional optical spectrum analyzer, clearly establishing the two-step transformation from time via frequency (wavelength) to space in the $800 \mathrm{~nm}$ spectral range. The wavelength dependence of the target's reflectivity is negligible as the bandwidth is less than $20 \mathrm{~nm}$ centered at $\sim 800 \mathrm{~nm}$.

events or presence of defects. High-speed laser scanners such as our scanner can address this predicament.

Specifically, we set up the HDLS in an interferometric configuration to monitor the surface profile of a fast-vibrating diaphragm (Figure 5a). As shown in Figure 5b, the axial position of each scanned point on the surface is encoded into the interferometer output (i.e., the interferogram in the time domain), retrieved from the interferogram and its Hilbert transform, and mapped into a 2D matrix for dynamical surface profilometry (See Methods). Figure $5 \mathrm{c}$ shows the frame sequence of a $1 \mathrm{kHz}$ nanomechanical surface vibration obtained by the HDLS-based surface vibrometer with a sub-nanometer axial resolution of $0.4 \mathrm{~nm}$ at a frame rate of $105.4 \mathrm{kHz}$. A movie of the diaphragm vibration is available (Supplementary Movie 1). The surface velocity profile of the vibrating diaphragm can also be obtained by differentiating the axial position of the surface profile with respect to time and is made possible by our scanner's fine temporal resolution of $9.5 \mu$ s (corresponding to $105.4 \mathrm{kHz}$ ). This is the first time that such a fast nanomechanical surface vibration was measured quantitatively in real time.
High-precision flow cytometry with the laser scanner. Finally, to demonstrate the biomedical utility of the HDLS, we applied it to flow cytometry for high-throughput screening of cells with high precision. Flow cytometry is an indispensable tool for counting and analyzing a large heterogeneous population of cells in pathology, immunology, and genetics. Unfortunately, the false positive rate and statistical error of conventional flow cytometers are high due to the lack of spatial resolution, rendering the system theoretically less able to resolve multiple cells (i.e., doublets, triplets, and clustered cells) within the interrogation beam (Figure 6a). The ability to provide spatial metrics (i.e., spectra in the spatial frequency domain) with an ultrafast laser scanner can be used as a simpler and more accurate method than other techniques ${ }^{32}$ to identify these events and hence reduce the false positive rate and statistical error. Specifically, the accuracy of the multiple-cell identification improves by a factor of the number of resolvable points or frequency bins in the spatial frequency domain $(\sim 1,000)$ provided by the HDLS divided by two frequency bins (i.e., forward and side scattering) provided by 

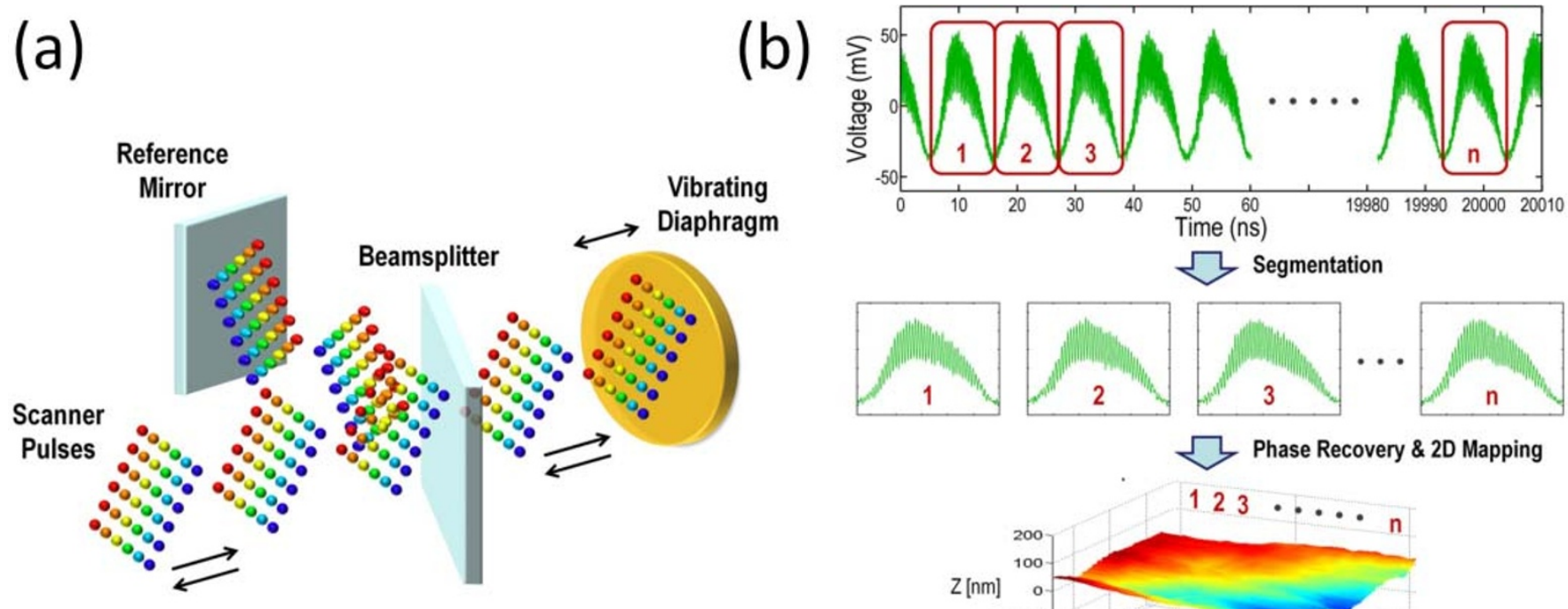

(c)

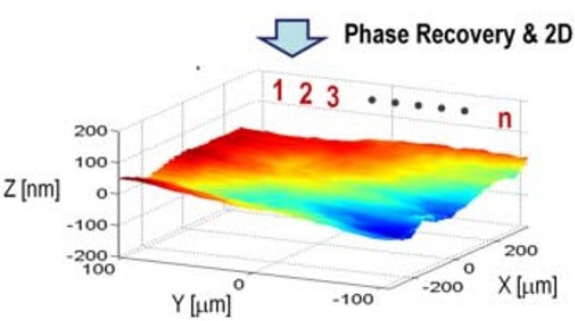

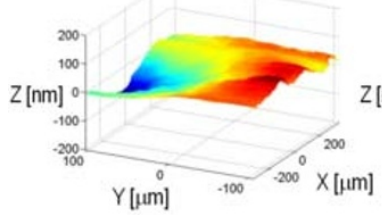

$t=0 \mu s$

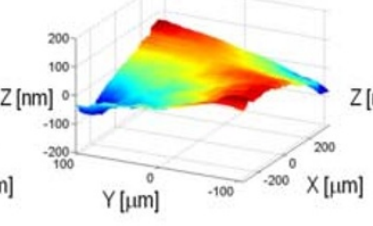

$t=95 \mu s$

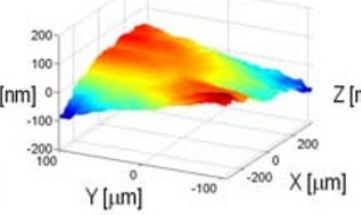

$\mathrm{t}=190 \mu \mathrm{s}$

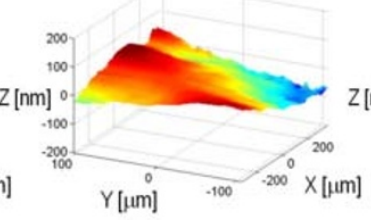

$t=284 \mu \mathrm{s}$

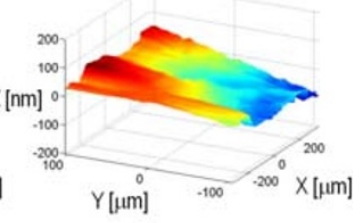

$\mathrm{t}=379 \mu \mathrm{s}$

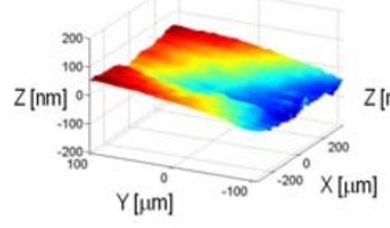

$\mathrm{t}=474 \mu \mathrm{s}$

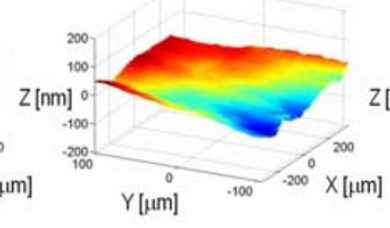

$\mathrm{t}=569 \mu \mathrm{s}$

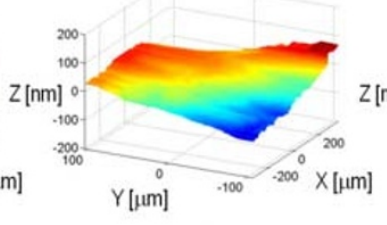

$t=664 \mu \mathrm{s}$

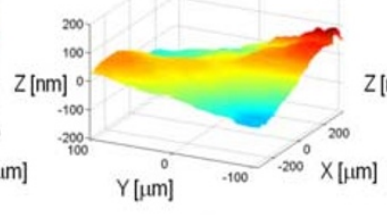

$\mathrm{t}=758 \mu \mathrm{s}$

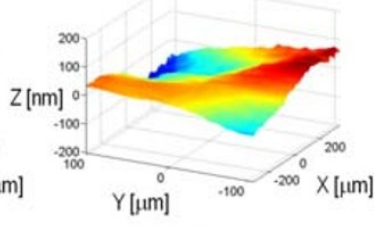

$\mathrm{t}=853 \mu \mathrm{s}$

Figure $5 \mid$ Surface vibrometry with the HDLS. (a) Experimental apparatus for the HDLS-based surface vibrometry. The HDLS is combined with a Michelson interferometer to quantitatively measure the axial position of the vibrating diaphragm at each different set of spatial coordinates.

(b) Procedure for producing time-resolved surface profiles with sub-nanometer axial resolution. The axial position of each scanned point on the surface is encoded into the interferometer output (i.e., the interferogram in the time domain), retrieved from the interferogram and its Hilbert transform, and mapped into a 2D matrix for dynamical surface profilometry. (c) Frame sequence of the $1 \mathrm{kHz}$ nanomechanical surface vibration captured by the HDLSbased surface vibrometer with a sub-nanometer axial resolution of $0.4 \mathrm{~nm}$ at a frame rate of $105.4 \mathrm{kHz}$ (corresponding to a temporal resolution of $9.5 \mu \mathrm{s}$ ). For clarity, only one every ten scans is shown. The complete frame sequence (movie) of the diaphragm's surface profile is available (Supplementary Movie 1). This is the first time that such a fast nanomechanical surface vibration was measured quantitatively in real time.

conventional flow cytometers. Alternatively, high-speed laser scanners can also be used to perform high-throughput examination of many individual cells without the need for a single stream of cells ${ }^{16,33}$.

To demonstrate HDLS-based flow cytometry, we constructed the experimental setup shown in Figure 6b. Here we employed inertial microfluidic technology ${ }^{33}$ to precisely position cells in flow with inertial lift forces while eliminating the need for sheath flow (Supplementary Information). Figure 6c compares size-based histograms of white blood cells and MCF7 breast cancer cells obtained by a conventional flow cytometer and the HDLS-based flow cytometer. It clearly indicates our method's ability to differentiate MCF7 cells from white blood cells as it can identify multiple white blood cells as false positive events. Consequently, the false positive rate and statistical error of flow cytometry were reduced by virtue of the complete spectrum in the spatial frequency domain provided by the HDLS's ultrafast laser-scanning capability, yet with high sensitivity and high throughput (Figure 6d). Our method is expected to be useful for high-precision size-based screening of rare cells in complex biological samples and high-throughput scanning of barcoded microparticles $^{34,35}$.

\section{Discussion}

In summary, we have proposed and demonstrated a new type of laser scanner that operates at nearly $100 \mathrm{MHz}$ - three orders of magnitude faster than conventional state-of-the-art laser scanners. This is made possible by employing spatiotemporal dispersion of broadband optical pulses, hence performing inertia-free laser scans without the need for mechanical and active electronic components. As a proof-of-principle demonstration, we have shown $1 \mathrm{D}$ line scans with 202 resolvable points at $90.8 \mathrm{MHz}$ and 2D raster scans with $202 \times 132$ resolvable points at $105.4 \mathrm{kHz}$. To show the utility of our ultrafast 
(a)

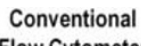

Flow Cytomete

Cytometer

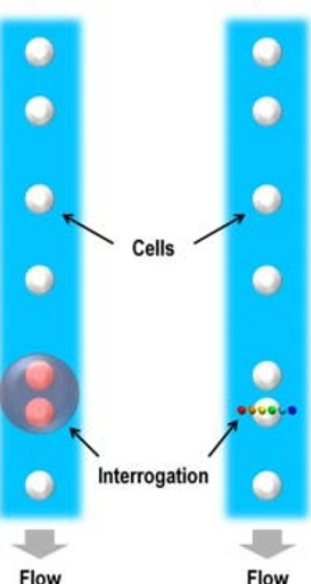

Flow Flow

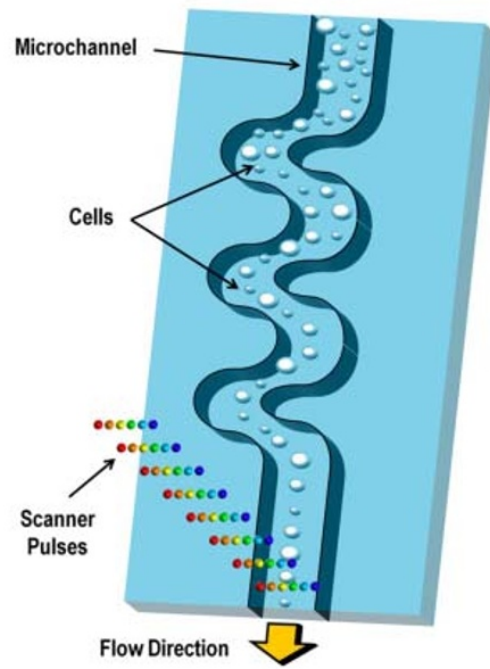

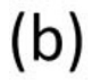

(b)
Conventional Flow Cytometer

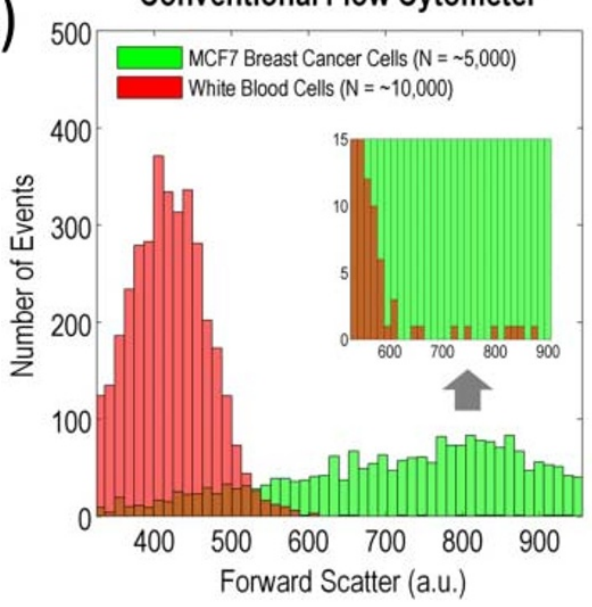

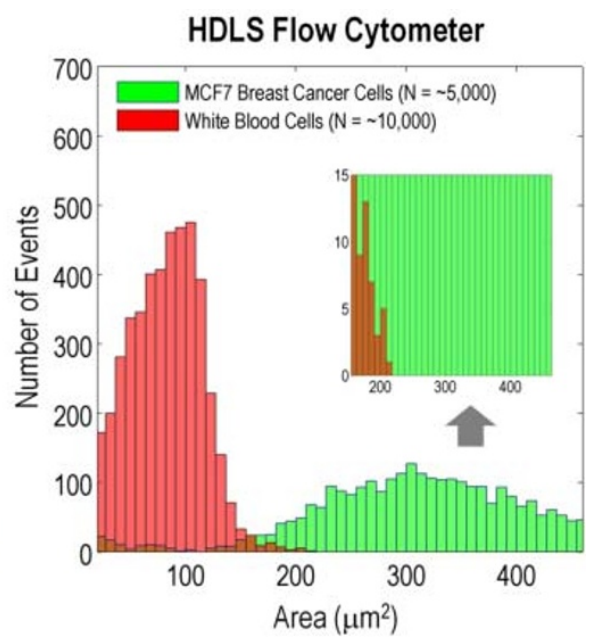

(d)

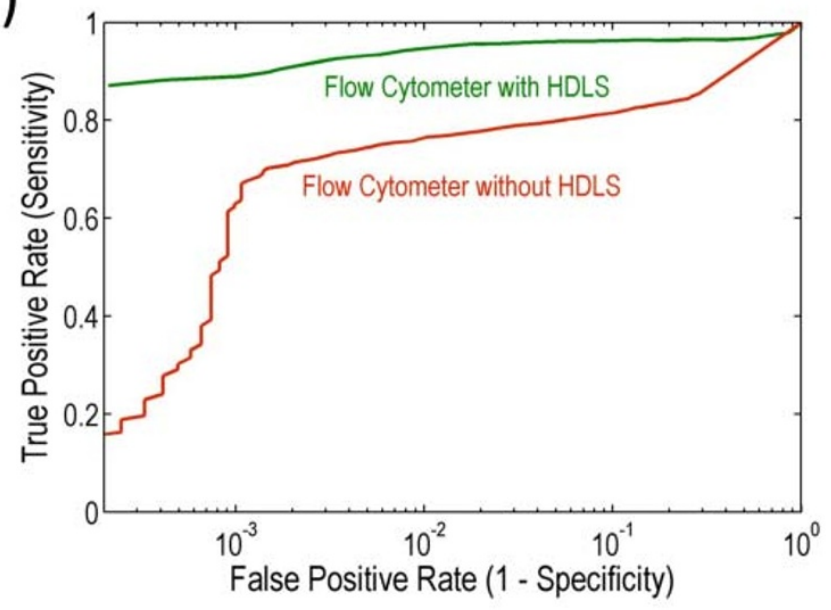

Figure 6 Flow cytometry with the HDLS. (a) Comparison in interrogation method between the conventional flow cytometer and HDLS-based flow cytometer. While conventional flow cytometers cannot resolve multiple cells (i.e., doublets, triplets, and clustered cells) within the interrogation beam due to the lack of spatial resolution, spatial metrics provided by the HDLS can be used to identify these events and hence reduce false positive rate and statistical error yet with high throughput. (b) Experimental apparatus for the HDLS-based flow cytometry. The microfluidic channel focuses and orders randomly distributed cells into a single stream with inertial lift forces (Supplementary Information). HDLS pulses are then focused onto the single stream of fast-flowing cells for forward-scattering measurements. Scattered light from the target is collected by an objective lens and detected by a single-pixel photodetector. To ensure stability, the microfluidic device was fabricated in thermoset polyester (TPE) using standard replica molding methods (Supplementary Information). The volumetric flow rate of cells was controlled by a syringe pump, and a uniform velocity of $1 \mathrm{~m} / \mathrm{s}$ was achieved by positioning cells precisely in a velocity gradient using inertial focusing. (c) Comparison of size-based histograms obtained with the conventional flow cytometer (left) and the HDLS-based flow cytometer (right) using identical samples of white blood cells and MCF7 breast cancer cells (measured separately). The figure clearly indicates our method's ability to differentiate MCF7 breast cancer cells from white blood cells as it can identify multiple white blood cells as false positive events. (d) Receiver operating characteristic (ROC) curve analysis showing that flow cytometry with the HDLS achieves lower false positive rate than flow cytometry without it, yet with high sensitivity and high throughput (Supplementary Information). This is made possible by the complete spectrum in the spatial frequency domain provided by the HDLS's ultrafast laser-scanning capability.

laser-scanning capability, we have used the HDLS to demonstrate ultrafast reflection imaging, real-time observation of nanomechanical surface vibrations, and flow cytometry with reduced false positive rate and statistical error.

In addition to three potential applications we have shown in this Article, our method can also be applied to a broad range of field applications in defense and manufacturing. Such applications include high-speed LIDAR for target tracking and law enforcement, high-throughput non-destructive evaluation and inspection, laser TV systems with high refresh rates, machine vision in robotics, and surface profilometry in the semiconductor industry. Moreover, the HDLS is also expected to be an effective tool for confocal microscopy and cytometry in advanced biological research and medical practice such as those found in pathology, histology, neurology, immunology, and microbiology. Finally, the HDLS is also applicable to non-industrial and non-biomedical settings including geographical survey, atmospheric science, art restoration, forestry, and archaeological study.

For sensing applications that employ weakly reflective objects and fluorescence detection, a few techniques can be concurrently used with the HDLS to improve sensitivity. First of all, the illumination beam can be made powerful by use of optical amplification ${ }^{36}$ in the dispersive fiber to compensate for reduced sensitivity. Since the pulses are stretched out due to the large GVD in the fiber so that the pulse train behaves as a quasi-continuous-wave field, they can be optically amplified by a large factor before target illumination. 
Moreover, sensitive detectors (e.g., photomultiplier tubes and avalanche photodetectors) can also be used for sensitive photodetection. For fluorescence detection, since the HDLS's bandwidth in our proof-of-principle demonstration is comparable the typical absorption bandwidth of fluorophores, their emission efficiency needs to be calibrated, depending on the spatial coordinates of the illumination on the target. To prevent a temporal cross-talk in the fluorescence signal between subsequent illumination points due to the ultrashort dwell time of the HDLS (51 ps in our demonstration), the rise of the fluorescence signal, which is much faster than the decay (typically longer than $1 \mathrm{~ns}$ ), can be used to locate the origin of the signal, provided that a fast sensitive photodetector is available.

\section{Methods}

Designer's equations for the HDLS. We discuss the "designer's equations" which predict the performance of the HDLS. Here the key parameters are the number of resolvable points, dwell time, and scan rate. Since these parameters are not independent, but interrelated, values for them need to be chosen carefully in order to optimize the performance of the scanner. First, the number of resolvable points $\left(n_{\mathrm{HDLS}}\right)$ is determined by the total optical bandwidth $(\Delta \lambda)$ and the spectral resolution limited by the temporal dispersion or the temporal analog of the far-field spectral resolution ${ }^{26}\left(\delta \lambda_{\tau}\right)$ as well as the spectral resolution limited by the spatial dispersion ${ }^{37,38}$ $\left(\delta \lambda_{S}\right)$. It is given by

$$
n_{\mathrm{HDLS}}=\Delta \lambda / \max \left(\delta \lambda_{\tau} \delta \lambda_{S}\right)
$$

where $\delta \lambda_{\tau}$ and $\delta \lambda_{S}$ are given by

$$
\delta \lambda_{\tau}=\lambda_{0} \sqrt{2 / c|D|} \text { and } \delta \lambda_{S}=\lambda_{0} \cos \theta / G d,
$$

respectively. Here we have assumed that the temporal and spatial dispersers are a dispersive fiber and diffraction grating, respectively. $c$ is the speed of light in vacuum, $\lambda_{0}$ is the center wavelength of the broadband pulse laser in vacuum, $D$ is the temporal dispersion or GVD, $\theta$ is the Littrow blaze angle of the incident beam with respect to the grating, $G$ is the groove density of the grating, and $d$ is the diameter of the beam incident on the grating. It is desirable to match the values of the two spectral resolutions to avoid any unnecessary dispersion. In our demonstration, they have comparable values based on the measured center wavelength $\left(\lambda_{0}=814 \mathrm{~nm}\right)$, optical bandwidth $(\Delta \lambda=17.5 \mathrm{~nm}), \mathrm{GVD}(D=-583 \mathrm{ps} / \mathrm{nm})$, groove density $(\mathrm{G}=2200$ lines/ $\mathrm{mm})$, beam diameter $(d=3 \mathrm{~mm})$, and Littrow blaze angle $\left(\theta=60^{\circ}\right)$, resulting in 202 resolvable points. Second, the time during which the HDLS dwells on each resolvable point or the so-called dwell time $\left(\tau_{\mathrm{HDLS}}\right)$ is found to be

$$
\tau_{\mathrm{HDLS}}=D \max \left(\delta \lambda_{\tau}, \delta \lambda_{S}\right) \text {. }
$$

In our demonstration, the dwell time is found to be $51 \mathrm{ps}$. Finally, the scan rate is equivalent to the pulse repetition rate of the broadband pulse laser. However, there is an upper limit on the scan rate $\left(R_{\mathrm{HDLS}}\right)$ to avoid overlaps of consecutive temporallydispersed pulses. The limit is given by

$$
R_{\mathrm{HDLS}}<R_{\max }=(D \Delta \lambda)^{-1} \text {. }
$$

In our demonstration, the limit on the scan rate is found to be $R_{\max }=98 \mathrm{MHz}$, which is higher than our scan rate $(90.8 \mathrm{MHz})$ and hence satisfies the requirement. All these equations are important for designing the HDLS and optimizing its performance.

Performance of the AOD. The number of resolvable points along the AOD's scan direction is given by $n_{\mathrm{AOD}}=\alpha B(1-\alpha / v T) / \xi v$, where $\alpha$ is the AOD's aperture, $B$ is the acousto-optic bandwidth, $v$ is the acoustic velocity, $T$ is the total frequency modulation scan time, and $\xi$ is the characteristic beam shape parameter ${ }^{19}$. In our proof-of-principle demonstration $(2 / T=105.4 \mathrm{kHz})$, the number is found to be $n_{\mathrm{AOD}}=132$ from $\alpha=6 \mathrm{~mm}, B=100 \mathrm{MHz}, v=4.2 \mathrm{~km} / \mathrm{s}$, and $\xi=1$.

Dispersion measurement. To measure the GVD of our dispersive fiber, we employed the method reported by Hult et $a^{\beta 9}$ which is based on a time-of-flight technique combined with a supercontinuum light source. It enables measurement of GVD with high spectral resolution over a large spectral range. The linear and quadratic fit functions were found to be $y=-(0.615 \mathrm{~ns} / \mathrm{nm}) x+(502 \mathrm{~ns})$ and $y=\left(0.000514 \mathrm{~ns} / \mathrm{nm}^{2}\right) x^{2}-(1.45 \mathrm{~ns} / \mathrm{nm}) x+$ (843 ns), respectively, where $x$ is the wavelength in units of $\mathrm{nm}$.

Reflection imaging with the HDLS. We derive the number of pixels in the target image. In the experiment, the back-reflected light from the target is detected via an optical circulator by a high-speed photodetector with $12 \mathrm{GHz}$ bandwidth. The output of the photodetector is digitized by a real-time digitizer with $16 \mathrm{GHz}$ bandwidth and $50 \mathrm{GS} / \mathrm{s}$ sampling rate. The number of pixels in the target image is found from the $\operatorname{GVD}(D=-583 \mathrm{ps} / \mathrm{nm})$, the sampling rate of the digitizer $\left(f_{\mathrm{dig}}=50 \mathrm{GS} / \mathrm{s}\right)$, the optical bandwidth $(\Delta \lambda=17.5 \mathrm{~nm})$, the pulse repetition rate $(R=90.8 \mathrm{MHz})$, and AOD scan rate $(r=2 / T=105.4 \mathrm{kHz})$ to be

$$
N_{H D L S} \times N_{A O D}=\left(D \times f_{\text {dig }} \times \Delta \lambda\right) \times(R / r)=510 \times 861 \text { pixels, }
$$

where $x$ and $y$ are the transverse axes orthogonal to the propagation axis of the incident light.

Surface vibrometry with the HDLS. We discuss the method for generating axially resolved surface profiles. To precisely measure the surface profile of the vibrating target, we employ Michelson interferometry in which the vibrating target and a fixed reference mirror are placed at the end of the interferometer's sample and reference arms, respectively (Figure 5a). Here the axial position of each scanned point on the surface is encoded into the output intensity of the interferometer (i.e., the interferogram in the time domain), which is given in the form of

$$
I(x, y, z)=I_{0}(x, y)+I_{1}(x, y) \cos \left[\phi_{0}+\phi_{1}(x, y, z)\right] .
$$

where $I_{0}(x, y)$ is the intensity profile of the incident light on the target, $I_{1}(x, y)$ is the intensity profile of the reflected light from the target, $\phi_{0}$ is the initial phase difference between the two arms of the interferometer, and $\phi_{1}(x, y, z)$ is the phase profile of the target. Here $x$ and $y$ are the axes in the transverse plane while $z$ is the axis along the axial direction. By introducing a relatively large phase shift between the two arms, the second term can vary much more rapidly than the first term. Then, high-pass filtering Eq. (6) removes the first term, yielding

$$
I_{\mathrm{HP}}(x, y, z)=I_{1}(x, y) \cos \left[\phi_{0}+\phi_{1}(x, y, z)\right] .
$$

Applying Hilbert transformation ${ }^{22}$ to the high-pass filtered interferogram generates

$$
H\left[I_{\mathrm{HP}}(x, y, z)\right]=I_{1}(x, y) \sin \left[\phi_{0}+\phi_{1}(x, y, z)\right] .
$$

Hence, the phase profile of the surface can be retrieved from Eqs. (7) and (8),

$$
\phi_{1}(x, y, z)=\tan ^{-1}\left\{H\left[I_{\mathrm{HP}}(x, y, z)\right] / I_{\mathrm{HP}}(x, y, z)\right\}-\phi_{0},
$$

and hence the axial displacement profile of the surface is given by

$$
d(x, y)=\phi_{1}(x, y, z) / k(x, y)=\lambda(x, y) \phi_{1}(x, y, z) / 2 \pi
$$

where $k(x, y)$ and $\lambda(x, y)$ are the wavenumber and wavelength of the incident light, respectively, and depend on the spatial coordinates of the interrogation point. In our proof-of-principle experiment, the axial resolution $(\delta z)$ is found to be $0.4 \mathrm{~nm}$ from the expression, $\delta z=2^{-\mathrm{b}} / k \sin 2 k\left(z_{0}+z\right)$, where $z_{0}$ is the initial offset to the sample arm length with respect to the reference arm length $\left(\phi_{0}=k z_{0}\right)$ and $b$ is the digitizer's improved effective number of bits (ENOB) $(b=8.3$ bits) (Supplementary Information). The number of pixels in the image of the vibrating target is the same as in the reflection imaging experiment $(510 \times 861$ pixels $)$.

Conventional flow cytometry. MCF7 breast cancer epithelial cells were harvested from culture and fixed by a 10 -minute incubation in $4 \%$ formaldehyde. Cells were washed and resuspended in PBS. Whole blood, obtained with an approved Institutional Review Boards protocol, was mixed with red blood cell lysis buffer (Roche) at a ratio of 2 (lysis buffer) to 1 (whole blood) and incubated for 10 minutes White blood cells were washed once in lysis buffer then resuspended in PBS. MCF7 and white blood cell suspensions were analyzed with the FACSCalibur system in the Flow Cytometry Core Laboratory at UCLA.

Flow cytometry with the HDLS. The microfluidic device was fabricated in thermoset polyester (TPE) (Supplementary Information). We used samples of white blood cells and MCF7 breast cancer cells identical to those used for analysis with the FACSCalibur system. The volumetric flow rate of cells was controlled by a syringe pump, and a uniform velocity of $1 \mathrm{~m} / \mathrm{s}$ was achieved by positioning cells precisely in a velocity gradient using inertial focusing. HDLS pulses are focused onto the cells in the microchannel via an objective lens with a numerical aperture of 0.4 . The $2 \mathrm{D}$ spatial metrics of cells were obtained by the combination of forward scattered light from 1D HDLS scans and the flow. The scattered light was collected via the same objective lens by the high-speed photodetector with $12 \mathrm{GHz}$ bandwidth, and the output signal was digitized by the real-time digitizer with $16 \mathrm{GHz}$ bandwidth and $50 \mathrm{GS} / \mathrm{s}$ sampling rate (identical to the detector and digitizer used in the experiment for Figure 4).

1. Marshall, G. F. Handbook of optical and laser scanning (Dekker, New York, 2009).

2. Fujii, T. \& Fukuchi, T. Laser remote sensing (CRC Press, New York, 2005).

3. Dotson, C. L. Fundamentals of dimensional metrology (Delmar Cengage Learning, New York, 2006)

4. Popescu, G., Ikeda, T., Goda, K., Best-Popescu, C. A., Laposata, M. L., Manley, S., Dasari, R. R., Badizadegan, K. \& Feld, M. S. Optical measurement of cell membrane tension. Physical Review Letters 97, 218101 (2006).

5. Gobel, W., Kampa, B. M. \& Helmchen, F. Imaging cellular network dynamics in three dimensions using fast 3D laser scanning. Nature Methods 4, 73-79 (2007).

6. Pawley, J. Handbook of biological confocal microscopy (Springer, New York, 2006).

7. Denk, W., Strickler, J. H. \& Webb, W. W. Two-photon laser scanning fluorescence microscopy. Science 248, 73-76 (1990).

8. Weitkamp, C. Lidar: Range-resolved optical remote sensing of the atmosphere (Springer, New York, 2005).

9. Schwartz, B. LIDAR: Mapping the world in 3D. Nature Photonics 4, 429-430 (2010).

10. Sinha, A. Vibration of mechanical systems (Cambridge University Press, Cambridge, 2010).

11. Pelesko, J. A. Modeling MEMS and NEMS (CRC Press, New York, 2002).

12. Osten, W. Optical inspection of microsystems (CRC Press, Boca Raton, 2006). 
13. Horn, B. K. P. Robot vision (MIT Press, Cambridge, 1986).

14. Hoffman, A., Goetz, M., Vieth, M., Galle, P. R., Neurath, M. F. \& Kiesslich, R. Confocal laser endomicroscopy: technical status and current indications. Endoscopy 38, 1275-1283 (2006).

15. Tarnok, A. \& Gerstner, A. O. Clinical applications of laser scanning cytometry. Cytometry 50, 133-143 (2002)

16. Vacca, G., Junnarkar, M. R., Goldblatt, N. R., Yee, M. W., van Skype, B. M. \& Briese, T. C. Laser rastering flow cytometry: fast cell counting and identification. SPIE Proceedings 7182, 71821T1-71821T10 (2009).

17. Mitsubishi Electric, http://www.mitsubishi-tv.com/laser.html

18. Conant, R. Micromachined mirrors (Springer, New York, 2002).

19. Goutzoulis, A. P. \& Pape, D. R. Design and fabrication of acousto-optic devices (CRC Press, Boca Raton, 1994).

20. Yaqoob, Z. \& Riza, N. A. Passive optics no-moving-parts barcode scanners. IEEE Photonics Technology Letters 16, 954-956 (2004).

21. Boudoux, C., Yun, S., Oh, W., White, W., Iftimia, N., Shishkov, M., Bouma, B., \& Tearney, G. Rapid wavelength-swept spectrally encoded confocal microscopy. Optics Express 13, 8214-8221 (2005).

22. Hult, J., Watt, R. S. \& Kaminski, C. F. High bandwidth absorption spectroscopy with a dispersed supercontinuum source. Optics Express 15, 11385-11395 (2007).

23. Asghari, M. H., Park, Y. \& Azana, J. Complex-field measurement of ultrafast dynamic optical waveforms based on real-time spectral interferometry. Optics Express 18, 16526-16538 (2010).

24. Wang, C. \& Yao, J. Complete characterization of an optical pulse based on temporal interferometry using an unbalanced temporal pulse shaping system. Journal of Lightwave Technology 29, 789-800 (2011).

25. Kelkar, P. V., Coppinger, F., Bhushan, A. S. \& Jalali, B. Time-domain optical sensing. Electronics Letters 35, 1661-1662 (1999).

26. Goda, K., Solli, D. R., Tsia, K. K. \& Jalali, B. Theory of amplified dispersive Fourier transformation. Physical Review A 80, 043821 (2009).

27. Chou, J., Boyraz, O., Solli, D. R. \& Jalali, B. Femtosecond real-time single-shot digitizer. Applied Physics Letters 91, 161105 (2007).

28. Goda, K., Tsia, K. K. \& Jalali, B. Amplified dispersive Fourier-transform imaging for ultrafast displacement sensing and barcode reading. Applied Physics Letters $\mathbf{9 3}$, 131109 (2008)

29. Goda, K., Tsia, K. K. \& Jalali, B. Serial time-encoded amplified imaging for realtime observation of fast dynamic phenomena. Nature 458, 1145-1149 (2009).

30. Siesler, H. W., Ozaki, Y., Kawata, S. \& Heise, H. M. Near-infrared spectroscopy: Principles, instruments, applications (Wiley, Weinheim, 2002).

31. Conway, J. A., Osborn, J. V. \& Fowler, J. D. Stroboscopic imaging interferometer for MEMS performance measurement. Journal of Microelectromechanical Systems 16, 668-674 (2007).

32. Donnenberg, A. D. \& Donnenberg, V. S. Rare-event analysis in flow cytometry. Clinics in Laboratory Medicine 27, 627-652 (2007).

33. Di Carlo, D. Inertial microfluidics. Lab on a Chip 9, 3038-3046 (2009)

34. Pregibon, D. C., Toner, M. \& Doyle, P. S. Multifunctional encoded particles for high-throughput biomolecule analysis. Science 315, 1393-1396 (2007).

35. Lee, H., Kim, J., Kim, H., Kim, J. \& Kwon, S. Colour-barcoded magnetic microparticles for multiplexed bioassays. Nature Materials 9, 745-749 (2010).
36. Goda, K., Mahjoubfar, A. \& Jalali, B. Demonstration of Raman gain at $800 \mathrm{~nm}$ in single-mode fiber and its potential application to biological sensing and imaging. Applied Physics Letters 95, 251101 (2009).

37. Yelin, D., Rizvi, I., White, W. M., Motz, J. T., Hasan, T., Bouma, B. E. \& Tearney, G. J. Three-dimensional miniature endoscopy. Nature 443, 765 (2006).

38. Tsia, K. K., Goda, K., Capewell, D. \& Jalali, B. Performance of serial time-encoded amplified microscope. Optics Express 18, 10016-10028 (2010).

39. Hult, J., Watt, R. S. \& Kaminski, C. F. Dispersion measurement in optical fibers using supercontinuum pulses. Journal of Lightwave Technology 25, 820-824 (2007).

\section{Acknowledgements}

We acknowledge support from the Microsystems Technology Office (MTO) in the Defense Advanced Research Projects Agency (DARPA), the Breast Cancer Research Program in the Congressionally Directed Medical Research Programs (CDMRP), and the National Institute of Health (NIH). K. Goda, C. Wang, and J. Adam are supported by the Burroughs Wellcome Fund, Natural Science and Engineering Research Council of Canada (NSERC), and German Research Foundation, respectively. The flow cytometry experiment was performed in the Jonsson Comprehensive Cancer Center (JCCC) and Center for AIDS Research Flow Cytometry Core Facility at UCLA that is supported by the NIH awards CA-16042 and AI-28697, and by the JCCC, the UCLA AIDS Institute, and the David Geffen School of Medicine at UCLA.

\section{Author contributions}

K. G. conceived the idea. K. G. and A. F. designed the optical layout for the HDLS. K. G. designed and performed the imaging experiment and A. F. performed digital image processing. K. G. and A. M. designed and performed the vibrometry experiment and A. M. performed digital signal processing. K. G. designed and K. G., C. W., Y. L. and R. B. performed the flow cytometry experiment and J. A. and A. A. performed digital image processing. D. R. G., E. S., O. M., E. C., Y. L., N. S. and R. B. provided materials for the experiments. K. G., D. R. G., D. D. and B. J. wrote the manuscript. D. D. and B. J. supervised the project.

\section{Additional information}

Supplementary information accompanies this paper at http://www.nature.com/ scientificreports

Competing financial interests: The authors declare no competing financial interests.

License: This work is licensed under a Creative Commons Attribution-NonCommercial-ShareAlike 3.0 Unported License. To view a copy of this license, visit http://creativecommons.org/licenses/by-nc-sa/3.0/

How to cite this article: Goda, K. et al. Hybrid Dispersion Laser Scanner. Sci. Rep. 2, 445; DOI:10.1038/srep00445 (2012). 